\title{
Laparoscopic repair of pericardio-diaphragmatic rupture: $A$ case report
}

\author{
M L Phakula, MMed, FCS (SA); M N Latakgomo, FCS (SA), FACS; A B van As, MBA, FCS (SA), PhD \\ Department of General Surgery, Pietersburg Hospital, University of Limpopo, Polokwane, South Africa
}

Corresponding author: M L Phakula (mlphakula@gmail.com)

Diaphragmatic rupture following blunt trauma is a common pathology which has good outomes when discovered early and treated. The common practice has been to manage these patients with either a laparotomy or thoracotomy, with some reports showing the safety of laparoscopy. We present a case of a patient who presented with a pericadio-diaphragmatic rupture who was managed successfully using laparoscopy.

S Afr Med J 2021;111(11b):1145-1146. https://doi.org/10.7196/SAMJ.2021.v111i11b.16194

Blunt diaphragmatic injury can be easily missed at initial presentation, especially in a setting without herniation through the defect. Patients who are diagnosed with diaphragmatic trauma need urgent surgery to prevent herniation of abdominal content or complications associated with herniation. The general management of these patients is a laparotomy or thoracotomy. There have been reports of laparoscopy used to treat these patients; however, this is not widely practised. We present a case of pericardio-diaphragmatic rupture which was managed successfully using laparoscopy.

\section{Case}

A 37-year-old male patient was brought to the emergency room as a referral from a district hospital following a motor vehicle accident. On arrival, his vital signs were as follows: blood pressure of 99/67, pulse of 91 , with a respiratory rate of 24 breaths per minute and oxygen saturation at $95 \%$ on face mask. He had a left intercostal drain inserted for a clinically diagnosed pneumothorax at the referring hospital. Examination of the cardiovascular system did not reveal any pathology. His Glasgow Coma Scale result was 14/15. $\mathrm{He}$ had multiple abrasions and some small lacerations which were not actively bleeding. Clinically there were no fractures noted. All parameters on his arterial blood gas were within normal ranges.

The chest X-ray (CXR) showed a loop of bowel in the left hemithorax with mediastinal shift to the right (Fig. 1). There were associated fractures of the 8 th to 10th ribs on the ipsilateral side. No brain injuries were noted on the brain computed tomography (CT) scan. A diagnosis of blunt diaphragmatic rupture with bowel herniation was made and the patient was referred to the surgeons. He was taken for laparoscopic diaphragmatic hernia repair.

Intraoperatively a pericardio-diaphragmatic rupture was discovered, and the stomach and transverse colon had herniated into the chest (Fig. 2). There was no bowel injury from the intercostal drain and there were no other intra-abdominal organ injuries. After reduction of the herniating contents, we proceeded to do laparoscopic repair of the injury. We used interrupted non-absorbable braided polyester suture (Fig. 3). He later developed a lung infection which was managed medically. He was discharged after 2 weeks in hospital after the lung pathology had cleared (Fig. 4).

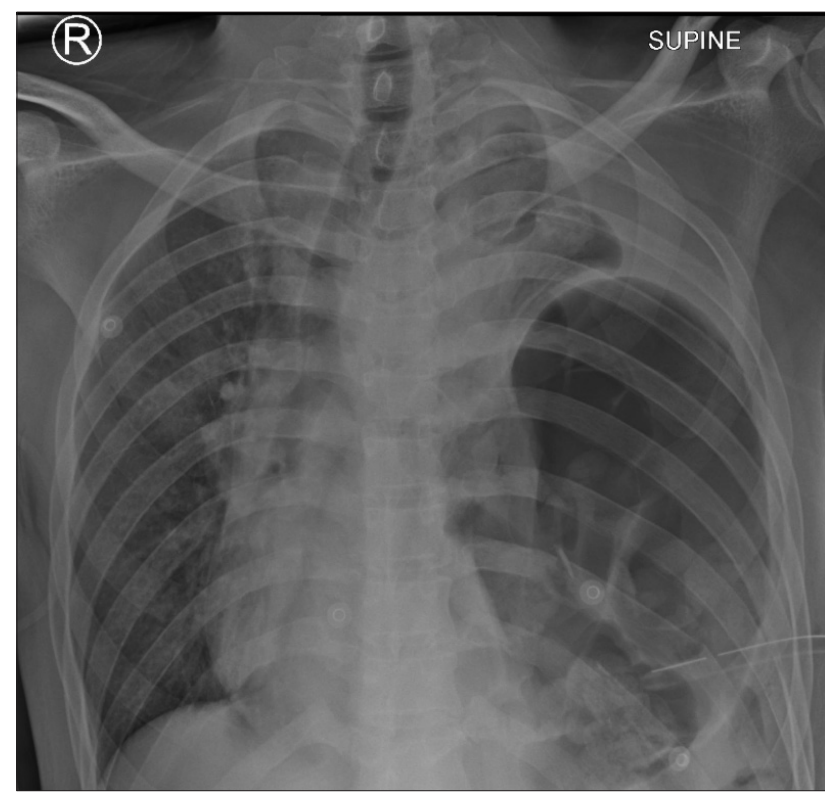

Fig. 1. Chest X-ray showing a loop of bowel in the left hemithorax with mediastinal shift to the right.

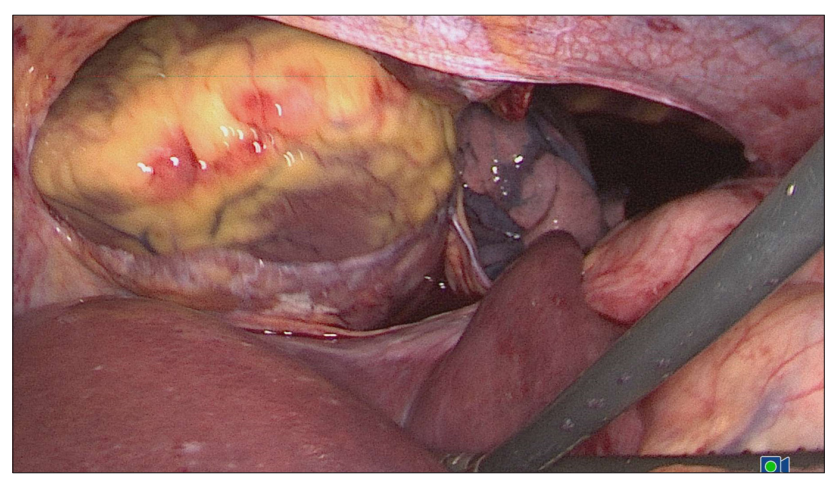

Fig. 2. Pericardio-diaphragmatic rupture with the stomach and transverse colon herniated into the chest. 


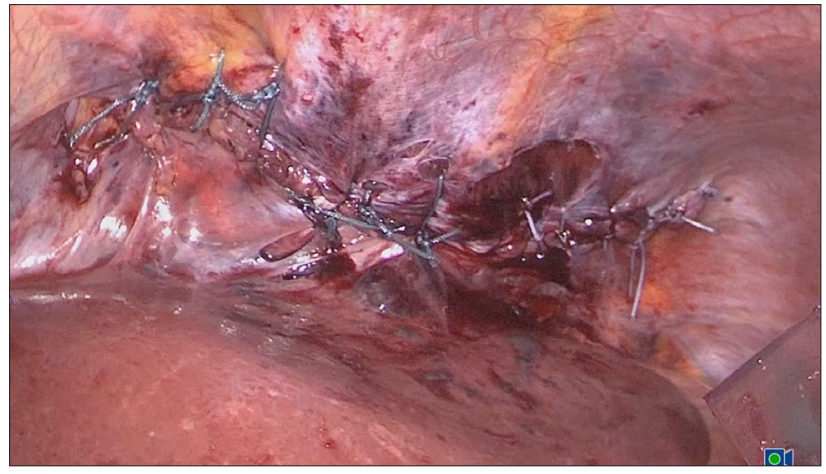

Fig. 3. Interrupted non-absorbable braided polyester sutures.

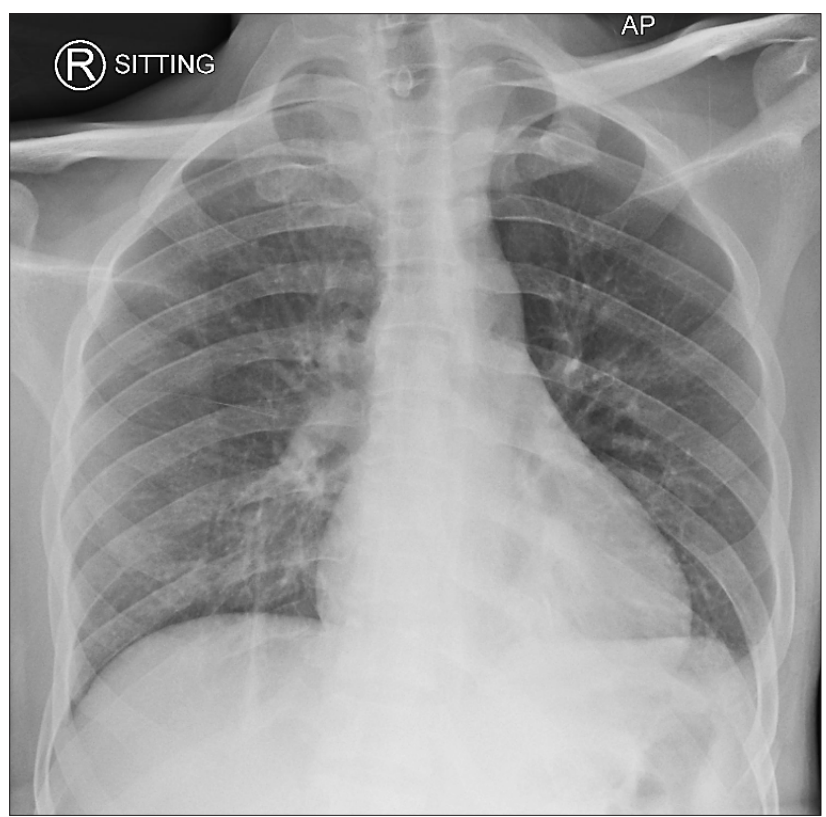

Fig. 4. Chest X-ray 2 weeks after surgery.

\section{Discussion}

Traumatic diaphragmatic injury (TDI) is a common condition which may easily be missed. It may result from both penetrating and blunt trauma with penetrating trauma responsible for the majority of cases. It is vital to have a high index of suspicion for TDI in patients with penetrating trauma to the thoraco-abdominal region. ${ }^{[1]}$ In the setting of blunt trauma, TDIs are usually associated with other injuries and may be picked up during investigations for the other injuries. There are no specific symptoms associated with diaphragmatic injury and, because diaphragmatic injuries can be associated with other injuries, they can easily be missed. Patients may present with difficulty in breathing and shock due to the associated injuries. On CXR, TDI may be easily identified when there is associated herniation of intraabdominal viscera into the chest. The presence of a nasogastric tube or an air-fluid level above the level of the diaphragm can be a diagnostic feature of this pathology. In the absence of herniation, however, the role of a CXR is limited in these patients. Just like the CXR, a CT scan has a high sensitivity and specificity for diagnosing diaphragmatic injury with herniation. It may also be used to determine the viscera which have herniated and other associated injuries. ${ }^{[2,3]}$ Both radiological investigations may miss right-sided diaphragmatic injuries because of the presence of the liver on the right, which usually prevents herniation in the acute phase. The challenge in managing diaphragmatic injuries becomes even more pronounced in the setting of an associated pericardial rupture. This may be suspected if pneumopericardium is noted on radiological imaging. However, in some cases, it may only be detected intraoperatively, as was noted with our case.

TDIs can be managed from either the chest or abdomen, depending on the surgeon's preference. In our institution we have adopted an abdominal approach for all patients with a clinical diagnosis or suspicion of TDI. A thoracic approach is used if there is an associated injury to intrathoracic organs that needs surgical intervention. An intra-abdominal approach allows for exploration of the abdomen and surgical management of associated organ injuries. ${ }^{[4]}$ Whether to repair the diaphragm open or laparoscopically depends on the available expertise. However, we believe that laparoscopy is a viable option for the management of these patients. We offer patients the benefits of laparoscopy, which include less postoperative pain and a shorter hospital stay, especially in the setting of an isolated diaphragmatic injury.

During the repair of the hernia, the goal is to approximate the edges of the defect in a tension-free manner. The recommended material is a non-absorbable suture, as was used in our patient. If approximation of the edges is not possible, then a mesh may be used, bearing in mind the potential complications associated with the use of a mesh in this setting. ${ }^{[5]}$

\section{Conclusion}

We present a case of a pericardio-diaphragmatic hernia which was repaired successfully using the laparoscopic approach. We propose that whenever the necessary expertise is available, such patients should be offered laparoscopic repair as a standard of care.

\section{Declaration. None.}

Acknowledgements. None.

Author contributions. MLP: Performed the surgery and managed the patient; wrote the manuscript. MNL: Co-managed the patient and assisted in writing the manuscript. ABvA: reviewed the manuscript and edited it before submission for publication.

Funding. None.

Conflicts of interest. None.

1. Fair KA, Gordon NT, Barbosa RR, et al. Traumatic diaphragmatic injury in the American College of Surgeons National Trauma Data Bank: A new examination of a rare diagnosis. Am J Surg 2015;209:864-869. https://doi.org/10.1016/j.amjsurg.2014.12.023

2. Turmak M, Deniz MA, Özmen CA, et al. Evaluation of the multi-slice computed tomography outcomes in diaphragmatic injuries related to penetrating and blunt trauma. Clin Imaging 2018;47:65-73. https:// doi.org/10.1016/j.clinimag.2017.08.010

3. Leung VA, Patlas MN, Reid S, et al. Imaging of traumatic diaphragmatic rupture: Evaluation of diagnostic accuracy at a level 1 trauma centre. Can Assoc Radiol J 2015;66:310-317. https://doi.org/10.1016/j. carj.2015.02.001

4. Hanna WC, Ferri LE. Acute traumatic diaphragmatic injury. Thorac Surg Clin 2009;19:485-489. https:// doi.org/10.1016/j.thorsurg. 2009.07.008

R. et al. Diaphragmatic rupture: Is management with biological mesh feasible? Int J Surg Case Rep 2012;3:349-353. https://doi.org/10.1016/j.ijscr.2012.04.011

Accepted 6 October 2021. 